\title{
CORRECTION TO: DIVISIBILITY PROBLEMS FOR FUNCTION FIELDS
}

\author{
S. BAIER ${ }^{1, *}$, A. BANSAL ${ }^{2}$ and R. K. SINGH ${ }^{1}$ \\ ${ }^{1}$ Department of Mathematics, Ramakrishna Mission Vivekananda Educational Research Institute, \\ G. T. Road, PO Belur Math, Howrah, West Bengal 711202, India \\ e-mails: email_baier@yahoo.de, rajneeshkumar.s@gmail.com \\ ${ }^{2}$ School of Physical Sciences, Jawaharlal Nehru University, New-Delhi 110067, India \\ e-mail: apabansal@gmail.com
}

(Received October 14, 2019; accepted November 14, 2019)

\begin{abstract}
In [3], we derived three results in additive combinatorics for function fields. The proofs of these results depended on a recent bound for the large sieve with sparse sets of moduli for function fields by the first and thirdnamed authors in [1]. Unfortunately, they discovered an error in this paper and demonstrated in [2] that this result cannot hold in full generality. In the present paper, we formulate a plausible conjecture under which the said three results in [3] remain true and the method of proof goes through using the same arguments. However, these results are now only conditional and still await a full proof.
\end{abstract}

Erratum to: Acta Mathematica Hungarica 156 (2) (2018), 435-448. DOI: $10.1007 / \mathrm{s} 10474-018-0853-4$

In [3], we derived the following three results in additive combinatorics for function fields.

THEOREM 1. We call a subset $\mathcal{S}$ of $\mathbb{F}_{q}[t]$ a $\mathcal{P}$-set if it consists of monic polynomials and no element of $\mathcal{S}$ divides the sum of two (not necessarily distinct) elements of $\mathcal{S}$ of larger degree. Let $\mathcal{S}$ be a $\mathcal{P}$-set of pairwise coprime polynomials in $\mathbb{F}_{q}[t]$ and denote by $\mathcal{A}_{\mathcal{S}}(N)$ the number of elements of $\mathcal{S}$ of degree not exceeding $N$. If $\varepsilon>0$, then there exist infinitely many positive integers $N$ such that

$$
\mathcal{A}_{\mathcal{S}}(N) \leq q^{N(1 / \Phi+\varepsilon)}
$$

where $\Phi$ is the golden ratio, given by

$$
\Phi:=\frac{\sqrt{5}+1}{2} .
$$

\footnotetext{
* Corresponding author.
} 
Theorem 2. If $\varepsilon>0, N \in \mathbb{N}$ is sufficiently large, $\mathcal{F}$ is a set of monic polynomials in $\mathbb{F}_{q}[t]$ of degree not exceeding $N$ and $f+f^{\prime}$ is square-free for all $f, f^{\prime} \in \mathcal{F}$, then

$$
\#(\mathcal{F}) \leq q^{N(2 / 3+\varepsilon)}
$$

Theorem 3. If $\varepsilon>0, N \in \mathbb{N}$ is sufficiently large, $\mathcal{F}, \mathcal{G}$ are sets of monic polynomials in $\mathbb{F}_{q}[t]$ of degree not exceeding $N$ and $f g+1$ is square-free for all $f \in \mathcal{F}$ and $g \in \mathcal{G}$, then

$$
\min \{\#(\mathcal{F}), \#(\mathcal{G})\} \leq q^{N(2 / 3+\varepsilon)} .
$$

The proof of these results depended on the following recent bound for the large sieve with sparse sets of moduli for function fields by the first and third-named authors in [1].

THEOREM 4. Let $Q$ and $N$ be positive integers, and $S$ be a set of nonzero monic polynomials in $\mathbb{F}_{q}[t]$ of degree not exceeding $Q$. Then

$$
\text { (1) } \sum_{f \in S} \sum_{\substack{r \bmod f \\(r, f)=1}}\left|\sum_{\substack{g \in \mathbb{F}_{q}[t] \\ \operatorname{deg} g \leq N}} a_{g} e\left(g \cdot \frac{r}{f}\right)\right|^{2} \leq\left(q^{N+1}+(\# S) \cdot q^{Q-1}\right) \sum_{\substack{g \in \mathbb{F}_{q}[t] \\ \operatorname{deg} g \leq N}}\left|a_{g}\right|^{2},
$$

where $a_{g}$ are arbitrary complex numbers.

For a generic set $S$, this is what one would expect, but unfortunately, the first and third-named authors discovered an error in this paper [1] and demonstrated in [2] that this result cannot hold in full generality. The counterexample provided there shows that one needs an additional factor of significant size. However, as far as this counterexample is concerned, this factor is bounded by $q^{\varepsilon(Q+N)}$. We therefore propose the following plausible conjecture which is a slight weakening of (1) and does not contradict the counterexample given in [2]. Except for particular cases, it seems to be difficult to establish this conjecture, though, and it is not clear if it holds in full generality or if some additional conditions on the set $S$ are required.

Conjecture 5. Let $\varepsilon>0$ be given. Let $Q$ and $N$ be positive integers and $S$ be a set of non-zero monic polynomials in $\mathbb{F}_{q}[t]$ of degree not exceeding $Q$. Then

$$
\sum_{f \in S} \sum_{\substack{r \bmod f \\(r, f)=1}}\left|\sum_{\substack{g \in \mathbb{F}_{q}[t] \\ \operatorname{deg} g \leq N}} a_{g} e\left(g \cdot \frac{r}{f}\right)\right|^{2} \ll_{q, \varepsilon} q^{\varepsilon(Q+N)}\left(q^{N}+(\# S) \cdot q^{Q}\right) \sum_{\substack{g \in \mathbb{F}_{q}[t] \\ \operatorname{deg} g \leq N}}\left|a_{g}\right|^{2},
$$

where $a_{g}$ are arbitrary complex numbers. 
Under this conjecture the Theorems 1-3 stated above remain true, and the methods of proof are unaffected. However, these theorems are now only conditional and still await a full proof.

\section{References}

[1] Stephan Baier and Rajneesh Kumar Singh, Large sieve inequality with power moduli for function fields, J. Number Theory, 196 (2019), 1-13.

[2] Stephan Baier and Rajneesh Kumar Singh, The large sieve with square moduli in function fields, arXiv:1910.05043.

[3] S. Baier, A. Bansal, and R. Kumar Singh, Divisibility problems for function fields, Acta Math. Hungar., 156 (2018), 435-448.

The online version of the original article can be found under DOI: $10.1007 / \mathrm{s} 10474-018-0853-4$ 